\title{
Un antropólogo en el aula: ¿Por qué los estudiantes se portan mal?
}

\author{
Wilmer Mejía Carrión \\ Universidad Nacional Federico Villarreal, Av. Nicolás de Piérola 355, Lima, Perú
}

Recibido el 15 de junio del 2017. Revisado el 22 de junio del 2017. Aceptado el 1 de julio del 2017

DOI: https://doi.org/10.33017/RevECIPeru2017.0007/

\section{Resumen}

La mala conducta de los estudiantes es una problemática común y constante, parece ser un mal endémico que parece no tener fin. La solución del problema se la ha encargado a un psicólogo(a), considerando que la disciplina de la psicología tiene la solución a este problema. Así pues, se considera que los comportamientos -malos o buenos- dentro del aula no competen a nadie más que a esta disciplina. ¿Qué podríamos hacer los antropólogos entonces dentro del aula más que solo enseñar algunos cursos de letras? En este trabajo se da cuenta de las conclusiones que un antropólogo hace a partir de un trabajo de campo en diversos colegios particulares. Dando una perspectiva diferente del comportamiento escolar dado por otras disciplinas. Se verán las relaciones de poder y la cultura dentro de la institución escolar y cómo estas afectan a los diversos actores sociales que operan en ella

Descriptores: Escuela, antropología, relaciones de poder, cultura escolar, educación

\section{Abstract}

Student misconduct is a common and constant problem, it seems to be an endemic trouble that seems to have no end. The solution of the problem has been entrusted to a psychologist, considering that the discipline of psychology has the solution to this problem. Thus, it is considered that the - bad or good - behaviors within the classroom do not compete with anyone else than this discipline. What could anthropologists do then in the classroom rather than just teach some courses of letters? In this paper I realize the conclusions that an anthropologist makes from a field work in several private schools. Giving a different perspective about school behavior given by other disciplines. Power relations and culture will be seen within the school institution and how these affect the various social actors operating in it.

Keywords: School, anthropology, power relations, culture, education

\section{Introducción}

8 a.m. Los estudiantes llegan presurosos al colegio. Hoy es lunes, único día en el cual se hace formación en el patio. ¡Firmes, descanso, atención! grita el auxiliar. Muchos de los estudiantes no hacen caso, siguen conversando, a pesar de los esfuerzos del auxiliar y un par de profesores. Al fin, estos logran, después de mucho esfuerzo, que todos hagan caso, encolerizado el auxiliar llama la atención de los alumnos vociferando ¿Por qué tratan así a sus autoridades?

La mala conducta de los estudiantes es una problemática común y constante, parece ser un mal endémico que parece no tener fin. -los alumnos no se portan bien, son terribles- dicen los profesores. Para paliar el problema y entender la problemática de los estudiantes, 
muchos colegios han instaurado un área psicopedagógica. Así de manera constante oen el peor de los casos- al menos un día a la semana un psicólogo o psicóloga trabaja en el colegio y entre otras labores, se encarga de evaluar al conjunto de estudiantes. Así, se busca resolver -0 al menos aminorar- el problema de la mala conducta del estudiantado.

Generalmente la culpa se achaca a la (mala) formación que han tenido en el hogar: padres muy autoritarios, madres demasiado blandas, familias disfuncionales de todo tipo. Sin contradecir ese diagnóstico pienso que hay un lado de la moneda que no es observado por las instituciones educativas: Que éstas mismas fomentan esta mala conducta.

¿La Antropología también puede abordar el problema?

Como se mencionó líneas arriba, la solución del problema se la ha encargado a un(a) psicólogo(a), considerando que la disciplina de la psicología tiene la solución a este problema. Así pues se considera que los comportamientos -malos o buenos- dentro del aula no competen a nadie más que a esta disciplina. ¿Qué podríamos hacer los antropólogos entonces dentro del aula más que sólo enseñar algunos cursos de letras?

En principio la herramienta principal de la antropología es la observación participante ya que:

"(...) puede descubrirse mucho observando directamente como se relacionan las personas recíprocamente (...) las personas están tan profundamente comprometidas recíprocamente que sólo podemos entenderlas de forma adecuada si interpretamos incluso sus nociones $y$ actitudes aparentemente íntimas como algo interpersonal" [1].

Esta herramienta puede aplicarse al aula de clases, gracias a ésta podemos entender que el estudiante no es un ente aislado sino que es parte de una categoría social y como tal tiene un status y rol dados complementarios al del docente.
Es aquí donde se forman pautas culturales las cuales señalaran como deben comportarse los individuos ante otros individuos que pertenezcan a otro grupo social. A este conjunto de pautas de conducta se le llama sistemas sociales. Ahora bien ningún grupo social se ajusta totalmente al sistema social es decir aunque se sabe cuáles deberían ser las conductas estas no se cumplen a cabalidad.

Como dice Linton:

"El funcionamiento de las sociedades depende de la presencia de pautas para la conducta recíproca entre individuos o grupos de individuos y es evidente que en tanto no haya intromisiones de fuentes externas más perfectamente ajustados a sus status $y$ funciones estén los miembros de cualquier sociedad, más fácilmente funcionará esta”. [2]

Partiendo de lo dicho, podemos afirmar que en el caso de los estudiantes y los profesores el colegio está organizado de tal manera que incentiva -sin quererlo- el mal comportamiento de los estudiantes ante las autoridades escolares. Ahora bien esta idea parece extraña ¿cómo es que una institución que se esfuerza tanto por conseguir encuadrar a los alumnos dentro de una serie de normas y reglas patea el tablero que ésta misma ha creado?

Se contestará a esta pregunta desde tres puntos de vista diferentes, en la primera se partirá por ver las relaciones entre docentes y estudiantes dentro del aula, destacando que está relación es una negociación entre ambos actores sociales, en el segundo se verá cómo las lógicas del mercado influyen en estas relaciones, esto es sobre todo válido para los colegios particulares, por último el tercer punto de vista se centrará en la cultura escolar y como está influye en la conducta de los estudiantes.

Cabe mencionar que los ejemplos dados del comportamiento de los alumnos se basan en observaciones realizadas en diversas instituciones educativas privadas en diferentes distritos de Lima.

\section{Metodología}


Para la realización de este trabajo he utilizado la técnica de la Observación Participante, llegando a tener una "participación activa" [3] pues estuve plenamente integrado en la estructura del colegio, los alumnos en muchas ocasiones me comentaron dentro de la clase lo malo y lo bueno de sus vidas como estudiantes.

Las observaciones fueron realizadas en tres centros educativos particulares, esto gracias a mi trabajo como docente del nivel secundario.

Estos colegios están ubicados en los distritos de Independencia, Ate y San Juan de Lurigancho. El tiempo de estas observaciones fue de cuatro meses (Independencia), de ocho meses (Ate) y siete meses (San Juan de Lurigancho). Mi trabajo -como docente- en estas instituciones educativas me permitió tener una mirada privilegiada de la relación entre alumnos y profesores.

Al ser yo docente, la relación con los alumnos fue directa, además que ellos se comportaron de la manera que siempre lo hacen pues para ellos yo era simplemente su profesor.

\section{Mirando las relaciones de poder}

\subsection{Salón de clases, sala de negociaciones.}

¿Qué es el poder?

a) Según Cohen, el poder es:

"una capacidad para influir en el comportamiento ajeno y/o influir en el control de las acciones importantes" [4]

El poder que tiene un profesor le es dado por las autoridades superiores a él, en este caso representados por la dirección del plantel, con el cual intenta hacer que los estudiantes se comporten de manera que se considere adecuada.

b) Es posible entender una institución escolar como el "terreno político" y el salón de clases como la "arena":

"Si bien es cierto que "los conceptos de terreno político y arena política siguen necesitando de una definición adecuada... otros le otorgan significados muy precisos. En este caso tomaremos los conceptos de F.G. Bailey que "considera que un terreno político es que en los grupos rivales, no comparten reglas convenidas de antemano para regular sus conflictos, la arena política como un área en la que los diversos contendientes aceptan las mismas reglas" (Ibídem, 119), además:

"Un terreno político no es más ni menos que el área de mayor actividad política definida por un investigador determinado., mientras que la arena es un área, dentro del terreno, en la que el investigador desea concentrarse en un momento dado" ( Ibídem, 119).

Se supone que el alumno debe cumplir con una serie de reglas que inclusive pueden estar escritas en su cuaderno de control, sin embargo para el buen funcionamiento de esta relación cada profesor dentro del aula tiene que poner las cosas claras de cómo va a ser llevada la clase desde el primer día que entra en el salón, para el mejor entendimiento de ambas partes.

\section{2 ¿Yo tengo el poder?}

Las jerarquías dentro de un colegio son claras, el profesor - junto con el auxiliar- ocupa un lugar subalterno dentro de él, como vemos en el siguiente cuadro ${ }^{1}$

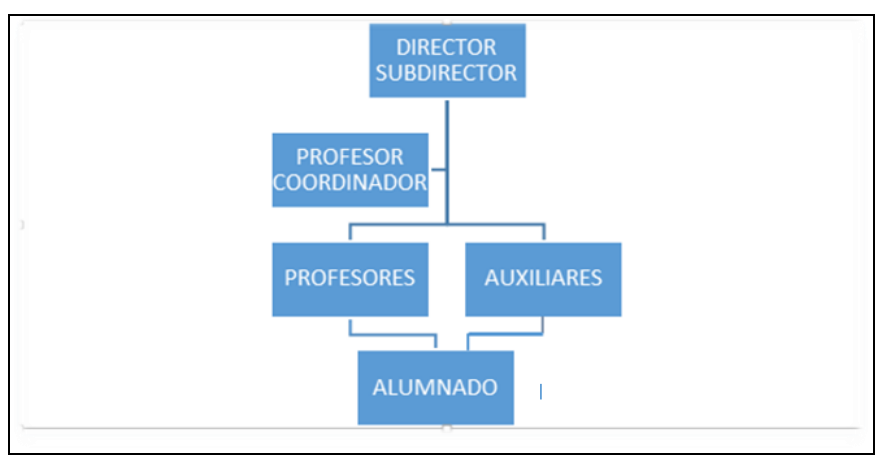

Figura 1: Organigrama Escolar. Elaboración propia

Ahora bien esto quiere decir que el profesor, si bien es cierto tiene autoridad sobre los alumnos está subordinado a las autoridades superiores quienes supervisan como es que el profesor enseña, esta supervisión no es directa en el sentido de que, no hay una

${ }^{1}$ Este es un esquema general que no intenta ser riguroso sino más bien didáctico 
intromisión en la clase del profesor, ni el director ni el subdirector pueden meterse a escuchar una clase pero sí lo pueden hacer mediante los alumnos, es decir el desempeño como profesor va asociado en gran medida a si los alumnos lo ven a uno como buen o mal profesor, lo cual influirá (aunque,-dependiendo el contexto- no sea totalmente concluyente) en la decisión de la dirección para continuar dentro de su staff de profesores.

El informe de desempeño laboral de un profesor puede tener diversas variables, apuntaré 4 de ellas:

a) Puntualidad

b) Dinamismo, solidaridad, lealtad en las actividades.

c) Empatía con los alumnos

d) Relaciones humanas profesor/padre de familia.

La empatía con los alumnos es importante pues es un referente directo al desempeño del profesor, por tanto los estudiantes tienen cierto poder, es aquí donde quiero detenerme y empezar a analizar la situación.

Primero deseo plantear esta idea, el profesor tiene una autoridad dada dentro del salón de clase pero necesita de legitimidad para poder hacer su trabajo de manera eficaz, vamos por partes, el profesor tiene un poder dependiente, este:

"...surge cuando un individuo con poder independiente, por su personalidad, por su cargo o por ambos, atribuye a otro el derecho de tomar decisiones. Puede hacerse de tres maneras: 1) Un individuo puede conceder poderes decisorios a otro; 2) un grupo que detente el poder puede asignar estos derechos a otros individuos; o 3) un grupo o individuo puede delegar esos derechos en otros individuos." (Ibídem, 121), "Por otro lado a pesar de eso su poder no se basa totalmente en la fuerza y la coerción.

Como nos dice Lewellen:

"La fuerza, por sí sola, es sin duda eficaz a corto plazo como medio de control político, pero si es la única base de aceptación popular pasiva, la sociedad será extremadamente rígida”. (Ibídem, 122)
Los profesores saben por experiencia esto, varias veces conversando con ellos me manifestaron que con los alumnos no se puede ir de frente "al choque" pues sería contraproducente.

Por lo tanto el poder del profesor de basa en una buena parte en el "consentimiento popular", por tanto podemos decir del "gobierno" dentro del aula tiene similar lógica que otro tipo de gobiernos, por ejemplo los del Estado, esto es así ya que:

"objetivamente un gobierno que posea poco poder consensual resultará demasiado rígido para llevar a cabo los ajustes necesarios para contentar a todo el pueblo; moralmente un gobierno de estas características tendría escasa justificación para una existencia prolongada." (Ibídem, 125)

Esto nos lleva al tema de la legitimidad, esta tiene bases distintas según las diversas culturas. Por ejemplo:

"un gran hombre" de la Polinesia puede conseguir la legitimidad mediante las mejores fiestas y los cerdos más cebados; un rey británico, lo consigue por su nacimiento dentro del linaje adecuado; un primer ministro, con el control de los votos parlamentarios; un jefe Cheyenne del siglo pasado, mediante su valor en la guerra, de joven, y con su sabiduría de viejo. Hay casos en la que la legitimidad no deriva en absoluto de la tradición, sino que debe lograrse de una manera totalmente nueva. Un dictador que llega al poder mediante una sublevación o un golpe de estado puede lograr legitimidad proporcionando ciertos beneficios a su pueblo." (Ibídem, 125)

¿En que se basa la legitimidad de un profesor dentro del aula? Definamos la legitimidad:

"La palabra legítimo significa legal, apropiado y que cumple ciertas reglas... La legitimidad se establece por tanto a través de cierto grado de consenso y acuerdo... la legitimidad se deriva de una combinación del consentimiento tácito y explícito de los individuos afectados del respeto a las leyes formales $y$ del reconocimiento por parte de otras entidades 
como Estados $y$ organismos internacionales."(Enciclopedia Encarta, 2006 Subrayado es mío)-

Podemos concluir que a nivel del aula lo más importante es el "consentimiento tácito y explícito de los individuos afectados" que en este caso serían los alumnos, es decir ellos deben aprobar las acciones del profesor dentro del aula, no solamente el profesor aprueba o desaprueba a los alumnos también lo hacen los alumnos hacia el docente, es decir que un factor principal para que seas considerado como un profesor está en el apoyo que obtengas de los alumnos.

\subsection{El alumno siempre sale ganando².}

Esta frase salió durante una charla que tuve con el sub director de un colegio en el cual estaba laborando como profesor, después de tener un problema de disciplina con tres alumnos del salón de 4to y 5to de secundaria. Estaba dictando clase cuando veía estos dos alumnos hacer demasiado bulla, constantemente les llamaba la atención y no me hacían caso, esto lo hicieron como tres veces, luego uno de ellos se sienta en otra carpeta-según por qué el otro le estaba fastidiando pero resulta que puso la carpeta mirando hacia los alumnos y teniendo a su espalda la pizarra, simbólicamente esa posición corresponde solo al docente, yo lo tome como una forma más de desacato, temiendo que mi autoridad se diluya lo volví a sentar junto al otro chico. Nuevamente empezaron a fastidiarse, no aguante más y los lleve a la subdirección.

Una vez allí, fue interesante como es que ellos usaron su poder en mi contra:

Primero socavaron mi legitimidad como docente, ya que parte la legitimidad de un

2 Las observaciones descritas en esta sección fueron hechas en un colegio particular en el distrito de Independencia el 2008. La etnografía completa está publicada en:

http://www.monografias.com/trabajos82/etnografiadel-colegio-san-luis-borja/etnografia-del-colegiosan-luis-borja.shtml profesor dentro de una clase se basa en el orden que puede mantener en el aula, un profesor que deje que en su clase haya desorden no es considerado un buen profesor, es por allí donde atacaron.

Yo dejé que dos alumnas jueguen porque no causaban mayor trastorno dentro del aula, ellas jugaban michi (tres en raya)-, pues estaba cada una en su lugar, mientras que ellos me causaban mucho mayor bulla.- Si pueden jugar les dije pero sin perturbar la clase-

- Ah, entonces podemos jugar" - dijo uno de ellos,- con ganas de fastidiar y empezó a jugar michi con el compañero de su costado, sentándose de tal manera que la pizarra y yo quedamos en segundo plano.

Había otro alumno que fomentaba el desorden, lo lleve a la subdirección y justo había venido su padre a ver como estaba su hijo, allí fue donde hablamos con él y empezamos los tres tratar de persuadirlo que el estudio era lo mejor para él, era interesante ver como para ese chico nuestras palabras eran eso, simples palabras sin mayor significado, ya que bien es cierto que escucho atento y sumisamente, no cumple con las tareas ni está al día en su cuaderno.

Cuando estábamos en la subdirección, empezaron a hablar que el desorden lo había fomentado yo, pues si dejaba que las alumnas jueguen ellos también podían hacerlo también. Yo argüí que deje que así era porque no me causaban mayor problema para continuar con la clase. El subdirector les increpó su conducta.

Al final lograron lo que querían, el subdirector me llamó la atención pues la acusación de que fomentaba el desorden era grave para un profesor - según el mismo me dijo-.Además me mencionó que algunas madres de familia se quejaron de que yo no hacía clase, es decir no hacía escribir a los chicos en el cuaderno y si bien es cierto explicaba esta no era la manera de enseñar a chicos de secundaria.

- "Menos mal que el chico no está al día" -me dijo- 
de tal manera que el docente sea sancionado, y así ser visto como abusivo.

\subsection{En apuros por un video}

Los pactos deben cumplirse, los docentes que incumplan con esto se verán sancionados por los alumnos, estos pactos no necesariamente son explícitos, es decir no necesariamente se verbalizan ni tampoco se escriben ${ }^{3}$.

Escribo un extracto de mi diario de campo para ejemplificar lo que digo:

"Hoy día vimos un video que trataba sobre el Cid campeador, el héroe nacional de España de la Edad Media. Ellos sabían que iban a ver videos así que apenas entre al salón, gritaron, ieh video, video! Incluso reclamaron cuando porque no traje inmediatamente DVD y el televisor. Ellos ven claramente que tienen todo el derecho de reclamar. (...) Mientras esperábamos deje que ellos conversaran y hablaran, en realidad no tenía otra opción, puesto que si quisiera mantenerlos absolutamente callados a todos sería contraproducente, puesto que no me harán caso, no lo verían legítimo, ya que yo desde la semana pasada les prometí el video y ellos lo esperaban, la espera los impacienta, no quieren saber nada de repasos, solo quieren ver el video. Yo mismo me sentí en ciertos apuros pues no traían los instrumentos y los chicos se decepcionarían de mí, y eso haría difícil el hacer clases pues se sentirían con todo el derecho de no hacerla, pues no cumpliría con ellos. Hubo algunos problemas con la disciplina, pero al final pudimos ver la película".

El aula se convierte en un espacio de encuentro de poderes de conflicto, el de los alumnos y el del profesor, los mismos profesores saben que no pueden poner total presión sobre los alumnos pues el alumno tiene una serie de derechos que si tal vez no son conscientes de ellos, - nunca he escuchado decir a un alumno, tengo mis derechos- si saben que pueden ejercer presión

\footnotetext{
${ }^{3}$ Lo cual no excluye lo contrario.
}

\section{Las lógicas del mercado.}

Aunque parezca contradictorio, muchos colegios particulares lejos de brindar el mejor servicio para atraer más estudiantes, lo que hacen es tratar de ahorrarse gastos, lo cual implica una baja calidad en el servicio y maximizar así las ganancias ${ }^{4}$. Esto sucede porque el colegio está inmerso dentro de una lógica de mercado en la cual los estudiantes son tratados como clientes y no como estudiantes Dentro de mi experiencia como docente en diversos centros educativos privados he podido constatar que los estudiantes se dan cuenta de esta situación y lejos de ser no pensantes -como muchos adultos los tildan-, se dan cuenta tanto de la situación en la que se encuentran y como de la falta de reciprocidad hacia el trato que se les da.

Un ejemplo claro de esta forma de pensar derivada de su desazón lo encontré en un estudiante que, en son de broma pero, quejándose dijo que la casa de su perro era más grande que su salón. Otro alumno en otra institución educativa me afirmó que sentía que en el colegio solo querían su plata. En otro aún, los estudiantes se daban perfecta cuenta que cuando los alumnos incurrían en alguna falta por más grave que fuese realmente nadie era sancionado y que el máximo castigoexpulsar a un alumno por un par de díashasta constituía un premio más que una sanción.

Por parte de los profesores existe otro problema, el sistema escolar privado no contrata a los docentes por tiempo completo sino que sólo los tiene por horas, esto hace que los docentes tengan que estar en dos o más colegios para poder conseguir ingresos aceptables. Así ciertos docentes no puedan ni

\footnotetext{
${ }^{4} \mathrm{Y}$ a pesar de eso siguen teniendo clientes, esto se debe en parte a que el desprestigio de la educación pública es tal que muchos padres de familia creen que prácticamente cualquier colegio particular es mejor que un colegio estatal, lo cual no es necesariamente cierto.
} 
recordar el nombre de todos sus alumnos ni puedan relacionarse de manera más cercana a sus estudiantes.

Otro de los problemas es que muchos colegios privados por no perder alumnos no son verdaderamente meritocráticos y los alumnos se dan cuenta de eso, lo cual genera incomodidad y desmotiva a los estudiantes.

\subsection{Masificación sin proyecto.}

Hay otro factor, el más importante pues de este derivan los expuestos anteriormente, a nivel nacional, la carrera magisterial esta desprestigiada. A pesar que el acceso a la educación creció desde inicios del siglo XX, reduciéndose la tasa de analfabetismo tanto en el campo como en la ciudad, esta masificación de la educación peruana se dio sin un proyecto que guíe sus pasos el resultado aumentó el acceso de la educación a sectores antes marginados pero de baja calidad. Con esto se dio una proliferación de instituciones educativas particulares - tanto a nivel de primaria, secundaria como superiorque tratan de llenar el vacío de una educación pública devaluada ya que el Estado peruano invierte poco en educación y esto ya es una crisis que tiene varios años.

Uno de los resultados de todo esto: El respeto hacia los docentes tanto de los alumnos como de los padres mismos es poco o nulo.

Esta idea esta tan acendrada que los mismos dueños de los colegios desautorizan al profesor restándole autoridad ante sus propios alumnos.

Este malestar creado por el sistema educativo y que los colegios refuerzan, sería la causa al menos en parte - de la mala conducta de los escolares. El estudiante siente que existe un desequilibrio en el trato que se le da. Aunque claro, salvo que sea un estudiante muy reflexivo, no se pondrá a pensar en el origen de sus sentimientos.

\section{5. ¿Por qué no se autorregulan? Cultura escolar y estudiantes}

Son las 10:30 am, el profesor está en plena clase, sin embargo el barullo y la bulla que hacen los alumnos conversando no dejan que el docente pueda realizar coherentemente su trabajo. Alza la voz, para tratar que sus palabras lleguen a todos. Cansado ya ni siquiera trata de acallarlos. En eso, otra profesora, que está enseñando en el salón contiguo, hija de la dueña del colegio, encolerizada aparece súbitamente en la puerta. Iracunda, vocifera. Que pasa con este salón, la bulla se escucha hasta la otra aula, ¿por qué no se autorregulan?

El sueño de todo docente es encontrar un salón de clases donde los alumnos estén atentos a las clases y no conversen entre ellos además de obedecer las reglas, siendo este un paraíso que pocas veces es visto por docente alguno, desde la antropología, no podemos hacer magia y hacer que los estudiantes se conviertan en obedientes y disciplinados seres atentos a toda la clase, sin embargo podemos entender los obstáculos que no permiten que los estudiantes no sigan las reglas.

En principio hay que entender algo, los docentes no solamente se "enfrentan" a individuos aislados sino que en realidad tratan con sujetos sociales es decir individuos dentro de grupos sociales que siguen normas y reglas sancionadas por este grupo. Así pues los alumnos no solamente se comportan como se comportan porque son inquietos "por naturaleza" sino que detrás de esos comportamientos se encuentra la aprobación de su grupo social.

Los sociólogos desde hace bastante tiempo atrás han dado una clasificación de los grupos humanos, mientras que los psicólogos sociales han hablado extensamente de como la pertenencia a estos grupos influye en la conducta de los individuos.

Así tenemos dos clases de grupos:

Grupo primario, grupo secundario, todo grupo social para funcionar necesita de un conjunto de reglas y normas, un sistema de ideas que guíe la conducta de sus miembros y sancione 
de manera positiva o negativa su comportamiento esto es cultura.

"La cultura consiste en pautas abstractas de y para vivir y morir. Estas pautas abstractas son culturales en la medida en que son aprendidas de manera directa 0 indirectamente en la interacción social y en la medida en que son parte de la orientación común de dos o más personas". [5]

Podemos decir entonces los grupos sociales humanos tienen y reflejan la cultura, pues bien entendemos que el colegio, es una institución social que alberga a diversos individuos los agrupa por edad en grados y secciones es decir en salones de clase, dentro de ellos los divide y subdivide, y así cada alumno va situándose poco a poco dentro de un grupo.

$Y$ cada uno de estos grupos va a formar sus propias normas y reglas, que a la vez estarán en mayor o menor relacionadas con las reglas y normas del colegio.

Me explicaré en términos antropológicos:

Cada salón será como una subcultura, y a las reglas y normas del colegio como parte de la gran cultura hegemónica que es la que supuestamente- le da las pautas a los individuos que se encuentran dentro de esta organización escolar, resultando cada salón como una subcultura dentro de estas, pero resulta que al no existir una cultura cohesionada se puede formar dentro del colegio salones ya no subculturales sino contraculturales.

Es decir que lejos de obedecer las reglas trataran de no hacerlo y sus miembros serán sancionados por el grupo primario como adecuado y hasta premiado entre sus pares.

Para entender esto de mejor modo, tenemos que internarnos no solamente lo que es cultura, sino específicamente lo que es cultura escolar, esto es importante porque:

"Cultura escolar, en efecto, puede significar muchas cosas diferentes e incluso superponerse 0 confundirse con otros conceptos"5

Así pues definamos lo que es:

“(...) la cultura escolar se podría definir como los patrones de significado transmitidos históricamente y que incluyen las normas, los valores, las creencias, las ceremonias, los rituales, las tradiciones, y los mitos comprendidos, quizás en distinto grado, por las personas miembros de la comunidad escolar Desde esta perspectiva, la cultura refleja distintos aspectos de una organización.

La organización tiene una cultura que puede ser funcional o disfuncional para sí misma (...)"6 (ibídem)

Quiero hacer énfasis en este último punto.

\subsection{Subculturas en la organización escolar ${ }^{7}$}

Debemos de conocer el término subcultura el cual a la vez servirá para entender la dinámica cultural dentro de una institución educativa.

En principio debemos entender que la cultura de las organizaciones escolares no siempre es coherente y compacta es decir que los actores sociales que forman parte de un centro educativo, no siempre actúan en consecuencia con sus símbolos, lemas o con lo que misión y visión que se expresan explícitamente.

Una subcultura agrupa a varias personas las cuáles comparten los mismos comportamientos y creencias o valores los cuales pueden distanciarse de la cultura dominante de su comunidad

Un salón puede tender a no sujetarse a las reglas dadas por la cultura escolar dominante.

\footnotetext{
${ }^{5}$ http://www.revistas.una.ac.cr/index.php/EDUCAR E/article/view/6591 consultado el 12/6/17 ${ }^{6}$ Ibídem

${ }^{7} \mathrm{Si}$ bien es cierto la organización escolar está compuesta por diversos actores sociales, me centraré en el alumnado. Espero concretar otro artículo donde escriba sobre la cultura escolar en la plana docente.
} 
Si los alumnos no se autorregulan, se debe en gran parte porque la subcultura del salón no está alineada a la cultura dominante.

No hay intereses que liguen a los estudiantes con la cultura escolar sino que más bien sus intereses estarán apuntando al de sus pares para que los aprueben, sin importarles demasiado las sanciones que pueden darle.

\section{Resultados y discusión}

La cultura escolar y la subjetividad de los estudiantes con respecto a las reglas y normas que se deben seguir en el colegio pueden estar en conflicto

"tanto la cultura como la subjetividad deben ser concebidas como realidades complejas y heterogéneas cuya dinámica está atravesada por la acomodación, la tensión y el conflicto" [6]

Así pues los estudiantes no van a sentirse parte de la cultura escolar porque simplemente lo diga el reglamento o cada lunes por la mañana se les recuerde a los alumnos que deben portarse bien.

Tampoco se van a portar bien por el simple hecho de que se les castigue continuamente, todo lo contrario en ocasiones los castigos pueden servir como una manera de prestigio dentro de su grupo primario. Por tanto los castigados pueden ser vistos como referentes a seguir o líderes.

Los estudiantes lejos de ser no pensantes, son bastante conscientes de la problemática escolar y de las relaciones de poder dentro de ella que pueden usar a su favor para socavar la autoridad del docente.

\section{Conclusiones}

Para los fines de este artículo hemos dividido la realidad escolar en tres partes:

a) Las relaciones de poder

b) Las lógicas de poder

c) Cultura escolar
Que están íntimamente relacionadas pues:

Las relaciones de poder dentro de una institución escolar están influidos por las lógicas del mercado y estás dos a la vez se enmarcan dentro de un entorno cultural que denominados cultura escolar.

Como se mencionó en la primera parte de este trabajo es importante entender que como todo grupo humano, las relaciones de poder, en este caso entre docentes y estudiantes debe existir un consenso. Una especie de pacto tácito donde se "negocien" los términos por los cuales se dé la obediencia a ciertas reglas dadas por una autoridad.

Estas relaciones se ven influenciadas por las lógicas mercantiles que la gran mayoría de colegios particulares han adoptado. La maximización de las ganancias no necesariamente se basa en la mejora de la calidad del servicio educativo.

Todo lo anterior se enmarca dentro de la cultura escolar, la cual muchos colegios no trabajan porque no son conscientes de la importancia de ésta para el mejor desempeño de todos los actores sociales que trabajan dentro de la institución educativa.

Se piensa que el problema es reciente, es frecuente escuchar que antes el alumno era más disciplinado sin embargo la historia nos da ejemplos de problemáticas de comportamiento escolar desde hace mucho tiempo atrás y que estos no se deben solamente a que los chicos son "rebeles por naturaleza" sino que se dan dentro de un marco de desorganización institucional.

Un historiador ha recogido diversos testimonios de la educación que se impartía en los colegios San Carlos y Nuestra Señora de Guadalupe durante el siglo XIX. Enfocándose en el primero escribe [7]:

“(...) la indisciplina de los alumnos carolinos. Ello se debía, en parte por la influencia del convulso ambiente político en los jóvenes 
carolinos pero sobre todo, por la inestabilidad económica y académica del colegio que no permitía implementar las medidas disciplinarias propias de un internado. Por ejemplo, la falta de mantenimiento en el local permitía que a través de forados o paredes bajas los alumnos salieran a la calle, o debido a la imposibilidad de darles de comer se les permitía ir a sus, casas. Los profesores mencionaban en 1827 que los alumnos estaban divididos en "bandos", tenían fugas del colegio, "tumultos" y la falta de respeto a sus superiores (...)"

Luego transcribiendo lo dicho en un documento de 1835 sobre el mismo colegio:

"(...) viven entregados al ocio, saliendo con frecuencia a la calle, toda hora de día y de noche, malgastando el tiempo que debieran llenar las tareas que les enseña su instituto (...) en lugar del recogimiento, la contracción y el estudio no hay más que abandono, insubordinación y excesos (...)"

¿Todo esto por qué? Eran los comienzos de la República, con una fuerte crisis económica que no permitía tener docentes de calidad y sanciones efectivas entre otras dificultades, que se reflejaban en la conducta de los estudiantes.

Sé que la problemática de la escuela es tan amplia, que probablemente el lector - sobre todo si es docente de carrera -que ha llegado hasta acá dirá que he dejado varios temas sin tratar. Admito eso, el problema de la escuela tiene tantas aristas que este pequeño artículo no puede abarcarlas todas.

Quiero concluir este trabajo con la siguiente reflexión:

Dejemos de pensar que son solamente los alumnos el problema, y dejemos de tachar de irracionales a aquellos a los cuales hemos puesto en un sistema escolar que incentiva la mala conducta.

\section{Agradecimientos}

Agradezco a los alumnos de las diversas instituciones educativas en las que trabajé, los cuales me enseñaron mucho a través de sus opiniones y comentarios.

\section{Referencias}

[1] M. Carrithers. ¿Por qué los humanos tenemos culturas?, 1 ra ed. (Alianza Editorial, Madrid, 2010) pp.27- 28

[2] R. Linton. Estudio del hombre, 1ra ed. (Fondo de Cultura Económica, México, 1942) p.122

[3] H. Russell, ed. Handbook on Methods in Cultural Anthropology, 1ra ed. (Altamira Press, United States, 1998) p.159

[4] T. Lewellen, Introducción a la Antropología Política, 2da ed. (Edicions Bellaterra, España, 2000) p.121

[5] H. Johnson, K. Young et al. Naturaleza, Cultura y Personalidad, 1ra ed. (Editorial Paidós: Buenos Aires, 1967) p.7

[6] G. Portocarrero y J. Komadina. Modelos de identidad y sentidos de pertenencia en Perú y Bolivia, 1ra ed. (IEP, Lima, 2001) p.15

[7] A. Loayza, D. Fernández y M. Garfías, eds. Trabajos de historia: religión, cultura y política en el Perú siglos XVII - XX, 1ra ed. (UNMSM, Lima, 2011). pp. 151 